American Journal of Applied Sciences 9 (8): 1242-1250, 2012

ISSN 1546-9239

(C) 2012 Science Publications

\title{
Distributed Cluster Based Routing Technique with Multiple Sinks for Wireless Sensor Networks
}

\author{
${ }^{1}$ Manisekaran, S.V. and ${ }^{2}$ R. Venkatesan \\ ${ }^{1}$ Department of Information Technology, Anna University of Technology, Coimbatore, India \\ ${ }^{2}$ Department of Information Technology, PSG College of Technology, Coimbatore, India
}

\begin{abstract}
Problem Statement: In order to reduce or minimize energy consumption and to improve bandwidth utilization in Wireless Sensor Networks (WSN), it was essential to consider its architecture in the network topology, power consumption, data rate and fault tolerance. Our objective is to formulate an effective cluster formation of nodes with multiple sinks to reduce power consumption and minimize the data loss. Approach: In this study, we propose to design a distributed cluster based routing technique in which multiple sinks were deployed. Initially the average distance between the sensor nodes and the sink was calculated and the nodes send their location information to the neighboring sinks. The sinks were updated this information at every time interval. The optimal sink places could be found using the global information based method. After sinking deployment, the information of each cluster was transmitted to other cluster using a gateway. The information gathered by the sink was transmitted to other clusters using a gateway. Results: By simulation results, we show that our proposed technique was enhanced the packet delivery ratio while reducing the energy consumption and delay. Conclusion: Our proposed approach minimizes the power consumption and data losses.
\end{abstract}

Key words: Wireless Sensor Networks (WSN), Cluster Head (CH), Cluster Based Hierarchical Routing Protocol (CBHRP), Distributed Cluster Based Routing (DCBR)

\section{INTRODUCTION}

Wireless Sensor Network (WSN): Densely deployed large number of nodes represents a sensor network. Each sensor node gathers the data and the information are sent back to the sink. (Balamurugan and Duraiswamy, 2011)WSN is a kind of Low Range Wireless Personal Area Network (LR-WPAN). (Ramakrishnan and Thyagarajan, 2009) Depending upon the application the nodes in the wireless sensor networks vary randomly (Kumar et al., 2009). The location information of the individual nodes is not known originally and thus there is a need of self organizing capability in the network. The major aspect of this network is the node cooperation and this is used to distribute the information gathered to their neighbor users. (Balamurugan and Duraiswamy, 2011) In military areas, natural disaster and health sensor networks play a major role. For the civil applications, light, temperature, humidity and other environmental factors are monitored in this network (Gandhi and Narayanasamy, 2011).

Clustering techniques in WSN: Clustering is used essentially in order to design a scalable sensor network protocols. In clustering, the nodes are divided into disjoint sets and using the Cluster Head $(\mathrm{CH})$, each node is placed on a chosen cluster. A standard and a high-level structure can be designed in the network using a clustering technique. Categorization of the clustered sensor networks is based upon the type and functionality of the nodes and this is discussed below:

(HevinRajesh and Paramasivan, 2012)

Homogeneous: All nodes have the same hardware and processing capabilities with cluster head being rotated periodically.

Heterogeneous: Capabilities and complex hardware are organized over the field together with numerous sensor nodes:

- The clustering techniques in WSN is quite advantageous for Distributed localized computing, fault-tolerance, load balancing, maximal network longevity, increased connectivity and reduced delay. But several issues are considered in clustering techniques such as (Chen et al., 2009)

- Proper management of the network deployment-In the fixed and random node deployment, entire area

Corresponding Author: Manisekaran, S.V., Department of Information Technology, Anna University, Coimbatore, India 
coverage and uniform energy consumption needs to be ensured by the network

- Unbalanced cluster head distribution-There are chances of unbalance, over load on neighboring nodes and node detachment in the heterogeneous network. This may occur when the cluster head intense on one part

- Uniform energy consumption-Balancing the energy dissipation and the reelection of the cluster head are required to balance the network energy consumption

- Network scalability-In order to cover more area or to extend the lifetime of the current network, new nodes have to be added and the clustering schemes should adapt to these changes

- Multi-hop or single hop communication-Energy can be consumed using proper and efficient communication. As concerned to the cost, the single hop communication is more expensive. On the other hand, in multi-hop communication more energy is lost since the nodes closer to the cluster head experience heavy traffic

HEED (Kour and Sharma, 2010) is a hybrid approach to select the cluster heads which is based on the residual energy and communication cost. In PEGASIS (Zarei et al., 2010) the close neighbors of each node only involve in communication. In ANTICLUST (Elbhiri et al., 2009), the cluster head selection is a two level process which involves communication between neighboring nodes.

Deploying multiple sinks: The amount of energy spent on communication in WSN can be decreased by diminishing the distance between sensors and sinks and also using the multiple sinks (Chen et al., 2009)

In large scale WSNs, common situations include multiple sources and sinks. When compared to the single sinks, better compatibility can be provided by the multiple sinks. Due to reduction in the multi-hop distance between sensor nodes and sinks, amount of energy consumption and the message transfer delay in communication are decreased effectively during the placement of the multiple sinks in proper locations. The path having the lowest hop count is considered to minimize the maximum worst case delay (Poe and Schmitt, 2009).

Problem identification and proposed solution: In our previous study (Manisekaran and Venkadesan, 2010), we have proposed an adaptive distributed clustering technique for reduction of battery power in WSN. It has two phases; a cluster formation phase and adaptive sleep duty cycle phase. In the cluster formation phase, the data generation rate and the similarity between data series is analyzed by the sink and based on these, the nodes are grouped into various clusters. In each cluster, the cluster heads are selected based on the connectivity and residual energy.

After the formation of clusters, in the sleep duty cycle phase, the data generation rates of cluster members are compared with a minimum threshold level. Finally these sleep/awake schedules are transmitted to each cluster members along with the cluster details by the sink. If a large proportion of nodes have data rate higher than the minimum threshold level, the similarity of their data is used. Then among such nodes, a fraction of nodes are provided with a sleep time mode alternatively in a distributed fair manner.

Each cluster head collects data from its members and check for any change in similarity or data rate and reported to sink. The sink then performs reclustering or rescheduling of sleep duty cycle, if necessary. Thus the workload of sink invested in checking of sensor data periodically is shared by the cluster heads, thus making our approach distributed.

There are certain drawbacks in this approach. The cost and energy of the route are not taken into consideration for inter-cluster and intra-cluster routing. More energy is consumed when the cluster head periodically sends the data and this leads to cluster head re-election. Due to that the clustering process is performed by a centralized sink, computational overhead can be caused. The entire clustering process is affected and this results in data loss.

Thus to overcome the drawbacks, we propose to design distributed clustering technique with multiple sinks for the wireless sensor networks. The average distance between the sensor nodes and the sink is calculated and the nodes send their location information to the neighbouring sinks. The optimal sink places can be found using global information based method. After sink deployment, the information of each cluster is transmitted to other cluster using a gateway. This technique enhances the packet delivery ratio while reducing the energy consumption and delay.

Related work: Erman et al. (2009) have presented a novel cross-layered communication protocol for efficient data dissemination in multi-sink WSNs. Their protocol combines network wide load balancing, clustering techniques and local routing optimizations with SENSEI architecture which make it efficient on both global and local level. They did not consider the impact of mobility of sensors and sinks.

Rashed et al. (2010) have proposed a two layer hierarchical routing protocol called Cluster Based Hierarchical Routing Protocol (CBHRP). They have 
introduced a new concept called head-set, consists of one active cluster head and some other associate cluster heads within a cluster. The head-set members are responsible for control and management of the network.

Akhtar et al. (2010) have presented an energy efficient routing technique named Energy Aware Intra Cluster Routing (EAICR). Their proposed technique has increased the network lifetime and number of packet sent in the network. They have shown that by using their technique, network lifetime has been increased up to $12 \%$ and energy consumption is reduced $17 \%$. They have also shown that by using their technique the number of data packets sent in the network has been increased up to $16 \%$.

Gupta and Dave (2009) have proposed architecture for reliable and real-time approach for data placement using sensor clusters. Their architecture suggest to store the information of all clsuters within a cell in the corresponding base station instead of storing information in individual cluster heads. For data dissemination and action they have used Action and Relay Stations (ARS).

Zarei et al. (2010) have proposed a novel cluster based routing protocol (CBRP) for prolong the sensor network lifetime. Their protocol achieves a good performance in terms of lifetime by balancing the energy load among all the nodes. In their protocol first the network is clustered using new factors and then a spanning tree is constructed for sending aggregated data to the base station which can better handle the heterogeneous energy capacities.

\section{MATERIALS AND METHODS}

\section{Proposed distributed cluster based routing technique with multiple sinks:}

Clustering technique: The clustering is performed based on the Similarity Measure Estimation which is explained in the previous study (Manisekaran and Venkadesan, 2010).

Multiple sink deployment:

Calculation of average distance between sensor nodes and the sink: A sink deployment technique is proposed which is mainly based on minimizing the energy consumption:

$\eta_{\mathrm{t}}=$ Energy demand of transmitting a packet to distance $\mathrm{k}$ apart

$\eta_{\mathrm{c}}=$ Energy that a sensor consumes to send a packet to 1 hop distance

$\mathrm{k}=$ Distance between the sensor and the nearest sink

$\mathrm{C}=$ Radio communication range of a sensor
If the packets are routed using a shortest path algorithm then $\eta_{\mathrm{t}}$ is given using following Eq. 1

$\eta_{\mathrm{t}} \sim\left[\frac{\mathrm{k}}{\mathrm{C}}\right] \times \eta_{\mathrm{c}}$

In a multi-hop network the energy cost of transmitting a message to the sink is linearly proportional to the distance the message has to travel. Thus, by minimizing the sum of the distances between the sensors and the sink, the energy spent for communication can be minimized.

Let $\mathrm{M}$ be the number of sinks and $\mathrm{H}$ denotes the number of sensor nodes. Consider sink $\mathrm{V}$ having coordinates $_{\mathrm{S}}(\mathrm{V})=\left(\mathrm{x}_{\mathrm{x}}^{(\mathrm{V})}, \mathrm{y}{ }^{(\mathrm{V})}\right), \quad \mathrm{v}=1, \ldots ., \mathrm{M}$. The distance vector from sensor ' $a$ ' to the $\operatorname{sink} l$ is represented using Eq. 2

$\mathrm{ka}=\sqrt{\left(\mathrm{x}^{(1)}-\mathrm{x}_{\mathrm{a}}\right)^{2}+\left(\mathrm{y}^{(1)}-\mathrm{y}_{\mathrm{a}}\right)^{2}}$

Unit vector pointing from the $a$ th sensor towards the $l$ th sink is shown using Eq. 3

$$
\mathrm{u}_{\mathrm{a}}^{(1)}=\frac{\mathrm{k}_{\mathrm{a}}^{(1)}}{\mathrm{k}_{\mathrm{a}}}
$$

This algorithm mainly focuses on minimizing the sensor's distance from the nearest sink which is represented using Eq. 4:

$$
\sum_{\mathrm{a}=1}^{\mathrm{H}} \operatorname{mink}_{1}(\mathrm{l}) \rightarrow \min
$$

The minimum distance to the sink can be obtained by setting the partial derivatives to zero which is represented using Eq. 5 and 6

$\frac{\partial}{\partial_{\mathrm{X}}(\mathrm{m})}\left(\sum_{\mathrm{a}=1}^{\mathrm{H}} \min _{1} \mathrm{k}_{\mathrm{a}}^{(1)}\right) \mathrm{s}_{0}^{(1)}, \ldots \cdots \cdot \mathrm{s}_{0}(\mathrm{~m})=0$,

$\mathrm{m}=1, \ldots \mathrm{M}$

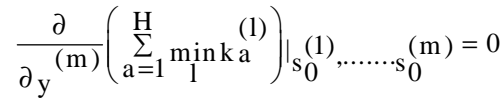

$\mathrm{m}=1, \ldots \mathrm{M}$

The partial derivatives are shown using Eq. 7-9:

$$
\begin{aligned}
& \frac{\partial}{\partial_{\mathrm{x}}(\mathrm{m})} \sum_{\mathrm{a}=1}^{\mathrm{H}} \min _{\mathrm{l}}(\mathrm{l})= \\
& \sum_{\mathrm{a}=1}^{\mathrm{H}} \frac{\partial}{\partial_{\mathrm{x}}(\mathrm{m})} \min _{\mathrm{l}} \mathrm{k}_{\mathrm{a}}^{(1)}
\end{aligned}
$$




$$
\begin{aligned}
& =\sum_{\mathrm{a}=1}^{\mathrm{H}}\left(\sum_{\mathrm{V}=1}^{\mathrm{H}} \frac{\partial}{\partial_{\mathrm{x}}{ }^{(\mathrm{h})}} \mathrm{ka} \cdot(\mathrm{V}) \cdot \mathrm{I}\left\{\mathrm{k}_{(\mathrm{a})}^{(\mathrm{V})}=\min 1 \mathrm{k}_{\mathrm{a}}^{(\mathrm{l})}\right\}\right) \\
& =\sum_{\mathrm{a}=1}^{\mathrm{H}}\left(\frac{\mathrm{x}^{(\mathrm{m})}-\mathrm{x}_{\mathrm{i}}}{\mathrm{k}_{\mathrm{a}}^{(\mathrm{m})}} \mathrm{I}_{\left\{\mathrm{k}_{(\mathrm{a})}^{(\mathrm{V})}=\min 1 \mathrm{k}_{\mathrm{a}}^{(\mathrm{l})}\right\}}\right) \\
& =\sum_{\left\{\mathrm{k}_{(\mathrm{a})}^{(\mathrm{V})}=\sum_{\operatorname{minlk}}^{(\mathrm{l})}\right\}}\left(\frac{\mathrm{x}^{(\mathrm{m})}-\mathrm{xi}_{\mathrm{i}}}{\left.\mathrm{k}_{\mathrm{a}}^{(\mathrm{m})}\right)}\right. \\
& \frac{\partial}{\partial \mathrm{y}^{(\mathrm{m})}} \sum_{\mathrm{a}=1}^{\mathrm{H}} \min _{\mathrm{l}}(\mathrm{k} \mathrm{a}= \\
& \mathrm{i}: \mathrm{k} a_{\mathrm{a}}(\mathrm{m}) \sum_{\min l \mathrm{ka}_{\mathrm{a}}}\left(\frac{\mathrm{y}^{(\mathrm{m})}-\mathrm{y}_{\mathrm{i}}}{(\mathrm{m})}\right)
\end{aligned}
$$

The requirements of (8) and (9) use the vectors and are represented as using Eq. 10

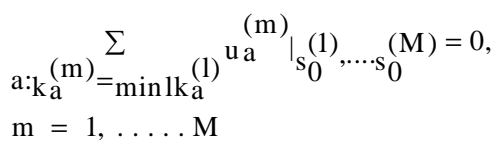

Let $\mathrm{C}_{\mathrm{V}}$ be the set of indices of the sensors closest to the sink $\mathrm{V}$ which is described in Eq. 11

$\mathrm{CV}=\left\{\mathrm{a}: \mathrm{ka}=\min _{\mathrm{k}}(\mathrm{l})\right.$

Here, $\left.C_{i} \cap C_{j}\right|_{i \neq j}=\lambda$ and $\cup_{i=1}, \ldots M C_{i}=\{1, \ldots H\}$ and the resultant vector of the orientation vectors of the sensors for sink $\mathrm{V}$ is shown using Eq. 12:

$$
\mathbf{r}_{\mathrm{v}}=\sum_{\mathrm{t} \in \mathrm{C}_{\mathrm{v}}} \mathrm{u}_{\mathrm{t}}^{(\mathrm{V})}, \mathrm{V}=1, \ldots \mathrm{M}
$$

When the resultant vector is zero for every sink, the average distance between the sensors and the closest sink will be minimal. (Vincze et al., 2007)

System model: We assume a sensor network which is densely deployed and is time driven in which the data message is sent for each time interval. The messages in the multi-hop communication network travel to the closest sink using the geographical routing.

Since no central authority is present in a distributed routing solution, end-to-end route is selected to give the information about the participating nodes. The decision taken for handing over the packet lies completely upon the nodes.

For routing process, a gateway is selected through which the data is transmitted to other cluster.
The mobile sink sends a periodical update messages which leads to the consumption of more energy but several factors are identified which can contribute to reduce power consumption. A broadcast message is used to cover up the entire region in the network and this updates the sensors directly. Dedicated power relay nodes are also deployed some times in order to forward the messages.

Before there is a change in the location of the sink, the data packets have to arrive at the sink. The update message includes a waiting time. The sink should stay at the broadcasted position until the waiting time. Data delivery can be assured within this waiting time. But if the data is not delivered within this time, relay nodes check whether the data arrived is late for last update message. If the data is late, then relaying node waits for certain time period so that it can be included in the next periodic update. Then packets are forwarded at the recently received sink position.

In this Fig. 1, we consider several sensors and sinks. For a multi-sink network, the sensors send their information about the location to their neighboring sinks. For few sensor nodes this information can be forwarded to the sink using the relay nodes R1, R2, R3 and R4. Four sinks are considered 1, 2, 3 and 4. Here the sensor nodes 1,2,3,4 and 5 transmit their location information to sink 1. Node 3 uses a relay node R1 to forward the message to the sink 1 . The sensor nodes $6,7,8,9$ and 10 are closer to sink 2 and thus forward their messages to the sink. Relay node R2 is used to forward messages from node 7 to the sink. The sensor nodes $15,16,17,18$ and 19 send their location information to sink 4 and the relay node R4 is used to forward the message to sink from node 15. Next, the sensor nodes 11, 12, 13 and 14 send their location information to the sink 3. Relay nodes check whether the packet to be forwarded is too late for the last update. In this the message transmitted from the sensor 11 is delayed and exceeds the waiting time for update. Thus the relay node waits and listens to the next periodic update (Vincze et al., 2007).

Global algorithm: The optimal sink places can be found using global information based method. We assume that the sinks have global knowledge, i.e., every sink knows the geographical coordinates of the sensors and the other sinks.

Given an initial sink setup, the sinks can decide which sensors are closest to them and divide the network into clusters, i.e., determine $\mathrm{C}_{\mathrm{V}}, \mathrm{V}=1$ : : :M. Next, the method uses an iterative procedure called determine centroid (Wagsta et al., 2001) to determine the centroids of the clusters, i.e., the places where sinks' resultant vector is zero. In every cluster, the sink calculates the resultant vector in beginning. 

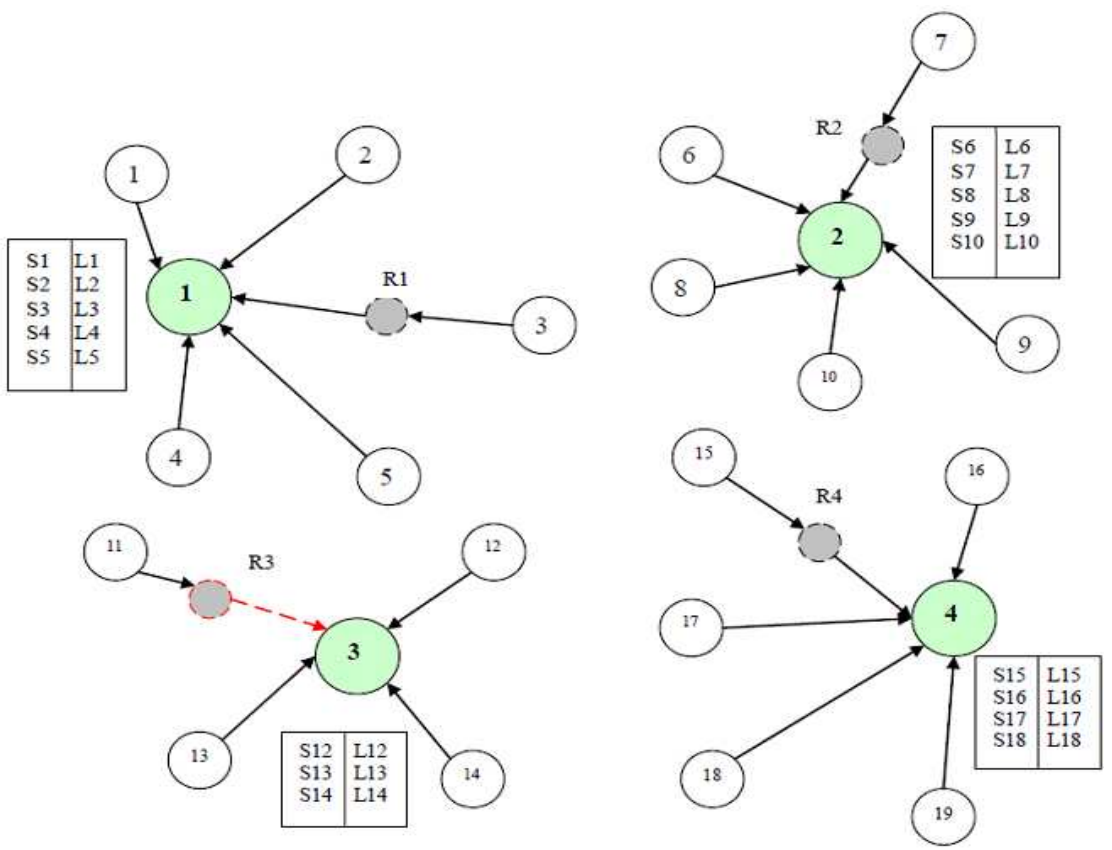

Fig. 1: Updating Location Information to Sinks

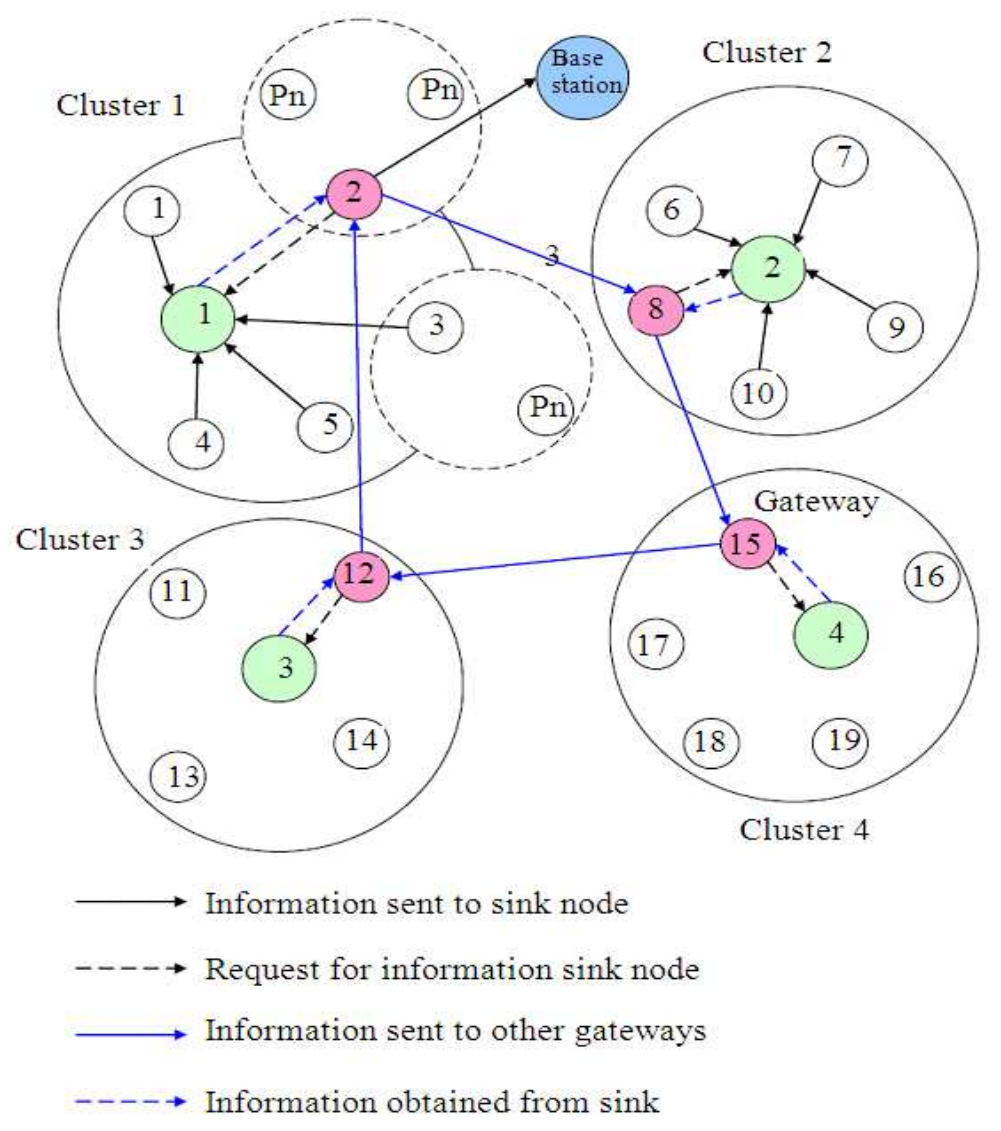

Fig. 2: Gateway selection and inter cluster routing 1246 
If $\mathrm{rv}$ is non-zero then it moves in the direction determined by the resultant vector, i.e., its new location is $\mathrm{s}^{(\mathrm{V})}+\mathrm{r}_{\mathrm{v}} \cdot{ }^{\text {MSP }}$ where MSP is the length of the maximal possible step for a sink. This iteration step is repeated until at least one of the sinks moves. As all the sinks have stopped the clusters are recalculated $\left(C_{V}^{\prime}\right)$ based on new sink locations. If there is a cluster that changed, i.e., $\mathrm{C}_{\mathrm{v}} \neq \mathrm{C}_{\mathrm{v}}$ ' for some $\mathrm{V}$ then the method returns to the determine centroid procedure to find the new centroids. The method stops if at the end none of the clusters is changed.

\section{Algorithm 1}

1. Initialize starting locations of the sinks.

2. Determine the clusters $\mathrm{CV}=\left\{\mathrm{a}: \underset{\mathrm{ka}}{(\mathrm{V})}=\min _{\mathrm{k}}(\mathrm{l})\right.$ $\mathrm{V}=1, \ldots \mathrm{M}$

3. Determine centroids.

4. For $\mathrm{j} \leftarrow 1$ to $\mathrm{M}$

$$
r V=\sum_{t \in C V} u t \text {, }
$$

$$
\begin{aligned}
& \begin{array}{l}
4.1 \text { If }\left|\mathrm{r}_{\mathrm{V}}\right|==0 \\
4.1 .1 \mathrm{~s}^{\mathrm{V}}=\mathrm{V}
\end{array} \\
& \text { else } \\
& \quad 4.1 .2 \mathrm{~s}^{\mathrm{V}}=\mathrm{s}^{(\mathrm{V})}+\mathrm{r}_{\mathrm{V}} \cdot \mathrm{MSP}
\end{aligned}
$$

end if

$$
\text { 5. If } \exists \mathrm{V} \in \mathrm{M}:\left.\right|_{\mathrm{rV}} \mid \neq 0
$$

then Determine centroids end if

6. Recalculating clusters with new sink locations

$$
\mathrm{CV}^{\prime}=\left\{\mathrm{a}: \underset{\mathrm{ka}}{(\mathrm{V})}=\min _{\mathrm{l}}{ }_{\mathrm{ka}}^{(\mathrm{l})}\right\}, \mathrm{V}=1, \ldots \mathrm{M}
$$

7. If $\forall \mathrm{V} \in \mathrm{M}: \mathrm{CV}==\mathrm{CV}$ then exit else

\section{End if}

Determine centroids

The sinks are aware of the locations of all sensors, therefore they can determine the final places of the sinks by running the algorithm without any real relocation of the sinks and thus the sinks have to be relocated once at the end of the algorithm. (Vincze et al., 2007).

Inter cluster routing: After the sinks are deployed in the network, the information of all the clusters needs to be transmitted to the base station. In order to find out the route from one cluster to another, a gateway is selected:
- Initially the $\mathrm{CH}$ broadcasts the advertisement message within its radio range and a primary node $(P n)$ receives it

- $\quad P n$ sends a joining request message to the $\mathrm{CH}$

- A beacon signal is generated by the $\mathrm{CH}$ within its radio range and the $\mathrm{Pn}$ which has heard the beacon becomes an ordinary node ( $\mathrm{Ln})$

- The farthest $\mathrm{Ln}$ from a $\mathrm{CH}$ is elected as a $\mathrm{GW}$ among one-hop neighbors of a $\mathrm{CH}$

- It as assumed that Pns are uniformly distributed, such that the farthest $\mathrm{Ln}$ from a $\mathrm{CH}$ will discover the largest number of Pns

- $\quad$ Each Ln among one-hop neighbors of a CH counts the number of Pns within its radio range and then, reports it to the $\mathrm{CH}$

- The Ln which discovers the largest number of Pns is elected as a GW by the CH. (Choi et al., 2005)

In this Fig. 2, the selection of gateway node for the cluster 1 is shown. Here the node 2 covers two initial nodes in its radio range and node 3 covers one initial node in its radio range. Thus node 3 is selected as the gateway node for the cluster 1. Accordingly, nodes 8, 12 and 15 are selected as gateways for the clusters 2, 3 and 4 respectively. After the selection of gateways, routing is performed through these gateway nodes. The information from the sensor nodes of cluster 1 are collected by the sink 1 and is given to the node 2 . Node 2 sends this information to the gateway node 8 of cluster 2. Node 8 transmits this information to the gateway node 15 of cluster 4 and node 15 transmits the information to the gateway node 12 of the cluster 3 . Finally the information is sent to the base station. Thus the routing is performed using the gateway nodes in this sensor network.

Complete algorithm: The steps involved in the entire process of distributed cluster based routing technique are summarized in the following algorithm.

Algorithm:

- Clustering is performed based on similarity measure estimation

- The distance between the sensors and the sink is calculated and their resultant vector is given in Eq. 12

- The resultant vector is zero for every sink, the average distance between the sensors and the closest sink will be minimal

- The locations of all the sensors are known by the sink 
- Sensor nodes transmit their location information to the nearby sink.

- The optimal sink places can be found using global information based method.

- The centroids of the clusters are determined using determine centroid method

- The distributed routing is provided to the network using gateway selection algorithm.

\section{RESULTS AND DISCUSSION}

Simulation setup: The performance of our Distributed Cluster Based Routing (DCBR) technique is evaluated through NS2 Network Simulator: www.isi.edu/nsnam/ns simulation. A random network deployed in an area of $500 \times 500 \mathrm{~m}^{2}$ is considered. Number of nodes is varied from 20-100. Initially the nodes are placed randomly in the specified area. The initial energy of all the nodes is assumed as 3.1 joules. In the simulation, the channel capacity of mobile hosts is set to the same value: 2 Mbps. The Distributed Coordination Function (DCF) of IEEE 802.11 is used for wireless LANs as the MAC layer protocol. The simulated traffic is CBR with UDP source and sink. The number of sources is varied as $5,10, \ldots .25$.

Table 1 lists the simulation parameters used.

Performance metrics: The performance of DCBR technique is compared with EEDC (Energy Efficient Dynamic Clustering) technique (Liu et al., 2007). The performance is evaluated considering the following metrics.

Average end-to-end delay: The end-to-end-delay is averaged over all surviving data packets from the sources to the destinations.

Average packet delivery ratio: It is the ratio of the number of packets received successfully and the total number of packets transmitted.

Energy consumption: It is the average energy consumption of all nodes in sending, receiving and forward operations.

\section{Simulation results:}

Based on nodes: In our initial experiment, we vary the number of nodes as $20,40,60,80$ and 100 , keeping the number of sources as 5 .

Figure 3 gives the average end-to-end delay when the number of nodes is increased.
Table 1: Simulation Parameters

\begin{tabular}{ll}
\hline No. of Nodes & $20,40, \ldots .100$ \\
\hline No. of sources & $5,10,15,20$ and 25 \\
Area size & $500 \times 500$ \\
Mac & 802.11 \\
Simulation time & $50 \mathrm{sec}$ \\
Traffic source & $\mathrm{CBR}$ \\
Packet size & 512 \\
Transmit power & $0.660 \mathrm{w}$ \\
Receiving power & $0.395 \mathrm{w}$ \\
Idle power & $0.335 \mathrm{w}$ \\
Initial enegy & $3.1 \mathrm{~J}$ \\
Transmission range & $75 \mathrm{~m}$ \\
\hline
\end{tabular}

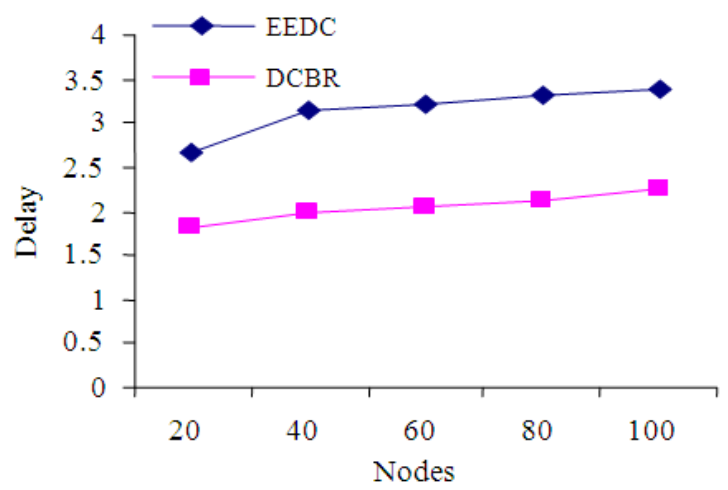

Fig. 3: Nodes Vs Delay

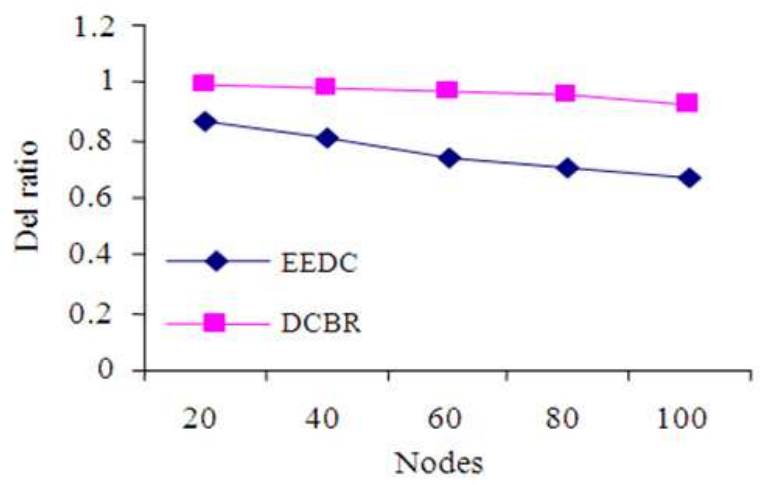

Fig. 4: Nodes Vs delivery ratio

From the figure, it can be seen that the average end-toend delay of the proposed DCBR technique is less when compared with EEDC. Figure 4 presents the packet delivery ratio when the number of nodes is increases. DCBR achieves good delivery ratio, compared to EEDC. Figure 5 shows the results of energy consumption when the number of nodes is increased. From the results, we can see that DCBR technique has less energy consumption when compared with EEDC, since it has the energy efficient tree. 


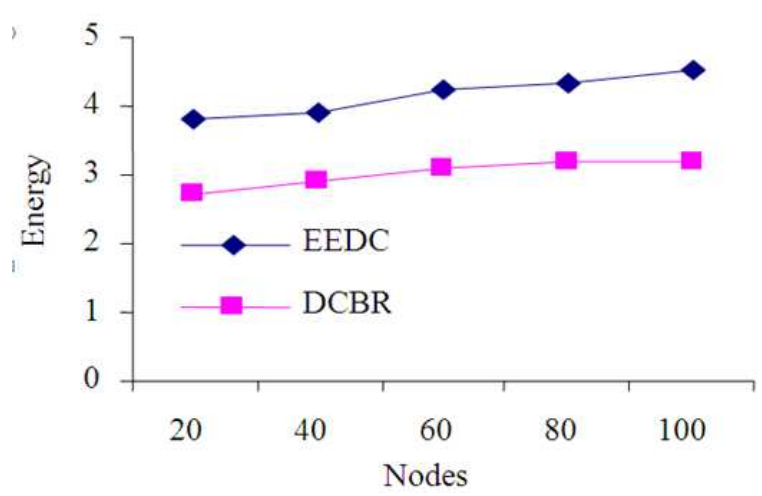

Fig. 5: Nodes Vs energy

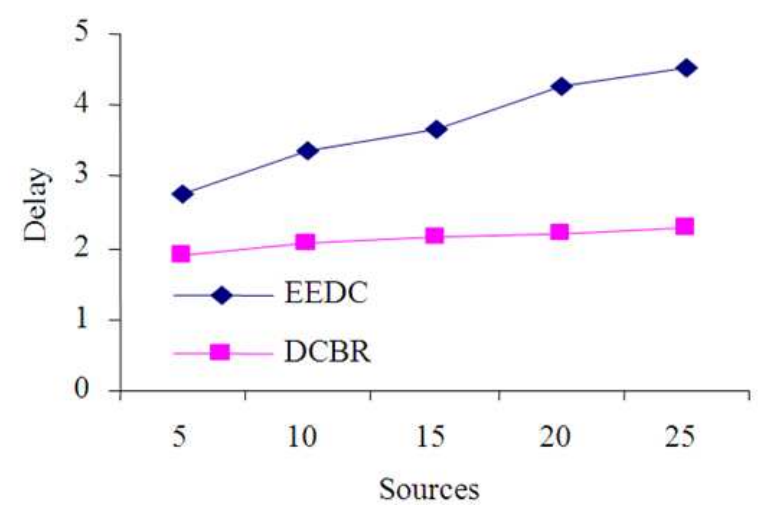

Fig. 6: Sources Vs delay

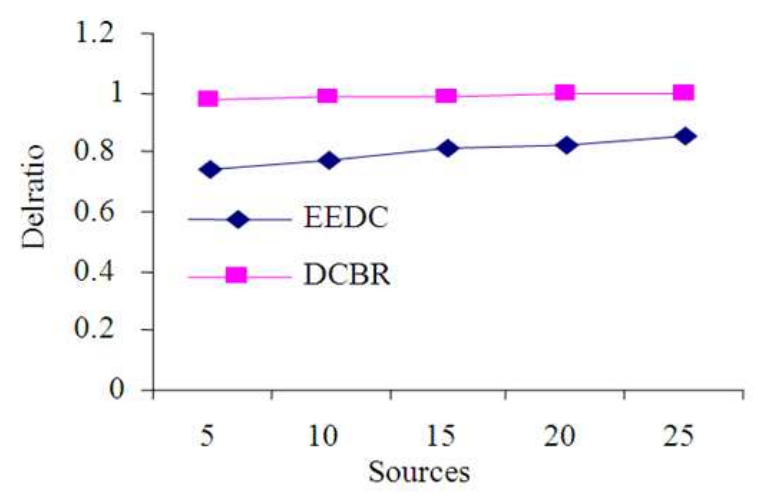

Fig. 7: Sources Vs delivery ratio

Based on sources: In the second experiment, we vary the number of sources as 5, 10, 15, 20 and 25, keeping the number of nodes as 100 .

From Fig. 6, we can see that the average end-toend delay of the proposed DCBR technique is less when compared with EEDC.

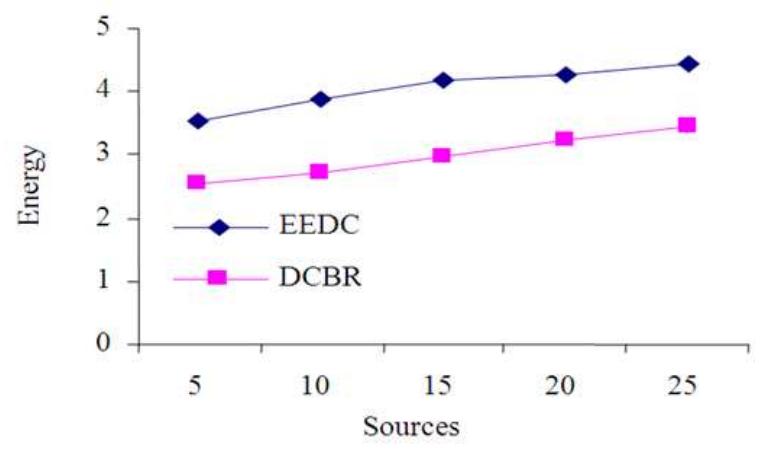

Fig. 8: Sources Vs energy

Figure 7 gives the packet delivery ratio when the number of sources is increased. DCBR achieves good delivery ratio, while being compared to EEDC. Figure 8 shows the results of energy consumption when the number of sources is increased. From the results, we can see that DCBR technique has less energy consumption against EEDC, since it has the energy efficient routing.

\section{CONCLUSION}

In this study, we propose to design a cluster based routing technique in which multiple sinks are deployed. Initially the average distance between the sensor nodes and the sink is calculated and the sensor nodes send their location information to the neighbouring sinks. The resultant vectors of the sensors are calculated. Centroid method is used to determine the places where sinks resultant vector is zero. The optimal sink places can be found using global information based method. The sinks decide which sensors are closest to them and divide the network into clusters. The gateway for each cluster is selected based upon the radio coverage of the sensor nodes in the cluster. Then the information of all the clusters is transmitted to other cluster using a gateway in each cluster. Information about all other clusters is collected in this way and is transmitted to the base station. By simulation results, we have shown that our proposed technique enhances the packet delivery ratio in addition to the reduction of energy consumption and delay.

\section{REFERENCES}

Akhtar, A., A.A. Minhas and S. Jabbar, 2010. Energy aware intra cluster routing for wireless sensor networks. Int. J. Hybrid Inform. Technol., 3: 29-47. www.sersc.org/journals/IJHIT/vol3_no1_2010/4.pdf

HevinRajesh, D. and B. Paramasivan, 2012. Fuzzy based secure data aggregation technique in wireless sensor. J. Comput. Sci., 8: 899-907. DOI: 10.3844/jcssp.2012.899.907 
Elbhiri, B., R. Saadane and D. Aboutajdine, 2009. Stochastic Distributed Energy-Efficient Clustering (SDEEC) for heterogeneous wireless sensor networks. ICGST-CNIR J., 9: 11-17.

Kumar, D., T.C Aseri and R.B. Patel, 2009. Analysis on deployment cost and network performance for heterogeneous wireless sensor networks. Int. J. Comput. Sci. Infor. Technol. (IJCSIT), 1: 109-120.

Zarei, B., M. Zeynali and V.M. Nezhad, 2010. Novel cluster based routing protocol in wireless sensor networks. Int. J. Comput. Sci. Issues, 7: 32-36.

Liu, C., K. Wu and J. Pei, 2007. An energy-efficient data collection framework for wireless sensor networks by exploiting spatiotemporal correlation. IEEE Trans. Parallel Distributed Syst., 18: 10101023. DOI: 10.1109/TPDS.2007.1046

Gandhi, K.I. and P. Narayanasamy, 2011. Mobile element scheduling for efficient data collection in wireless sensor networks: A survey. J. Comput. Sci., 7: 114-119. DOI: 10.3844/jcssp.2011.114.119

Manisekaran, S.V. and R. Venkadesan, 2010. An Adaptive Distributed Power Efficient Clustering Algorithm for Wireless Sensor Networks American J. Sci. Res., 10: 50-63.

Balamurugan, P. and K. Duraiswamy, 2011. Consistent and proficient algorithm for data gathering in wireless sensor networks. J. Comput. Sci., 7: 14001406. DOI: 10.3844/jcssp.2011.1400.1406

Erman, A.T.U., T. Mutter, L.V. Hoesel and P. Havinga, 2009. A cross-layered communication protocol for load balancing in large scale multi-sink wireless sensor networks. Proceedings of the 9th International Symposium on Autonomous Decentralized Systems, Mar. 23-25, IEEE Xplore Press, pp: 1-8. DOI: 10.1109/ISADS.2009.5207344

Poe, W.Y. and J.B. Schmitt, 2009. Self-Organized Sink Placement in Large-Scale Wireless Sensor Networks. Proceedings of the 17th Annual Meeting of the IEEE International Symposium on Modeling, Analysis and Simulation of Computer and Telecommunication Systems, MASCOTS, Sept. 21-23, IEEE Xplore Press, Germany, pp: 1-3. DOI: 10.1109/MASCOT.2009.5366741
Ramakrishnan, S. and T. Thyagarajan, 2009. Energy efficient medium access control for wireless sensor networks. IJCSNS Int. J. Comput. Sci. Network Secu., 9: 273-279.

Rashed, M.G., M.H. Kabir, M.S. Rahim and S.E. Ullah, 2010. Cluster based hierarchical routing protocol for wireless sensor network. Int. J. Comput. Network Secu., 2: 5-5.

Chen, G., C. Li, M. Ye and J. Wu, 2009. An unequal cluster-based routing protocol in wireless sensor networks. J. Wireless Networks, 15: 193-207. DOI: $10.1007 / \mathrm{s} 11276-007-0035-8$

Wagsta, K., C. Cardie, S. Rogers and S. Schroedl, 2001. Constrained k-means clustering with background knowledge. Proceedings of the 18th International Conference on Machine Learning, (ML' 01), Cornell University, Ithaca, New York, pp: 577-584.

Choi, J.Y., C.G. Kang, Y.S. Kim and K. Hur, 2005. Neighbor node discovery algorithm for energyefficient clustering in ubiquitious sensor networks. Proceedings of the 2005 International Conference on Embedded and Ubiquitous Computing, (EUC' 05), Heidelberg, pp: 470-479. DOI: 10.1007/11596042_49

Kour, H. and A.K. Sharma, 2010. Hybrid energy efficient distributed protocol for heterogeneous wireless sensor network. Int. J. Comput. Applications, 4: 1-5.

Gupta, S. and M. Dave, 2009. Distributed real time architecture for data placement in wireless sensor networks. The Pennsylvania State University.

Vincze, Z., R. Vida and A. Vidacs, 2007. Deploying multiple sinks in multi-hop wireless sensor networks. Proceedings of the IEEE International Conference on Pervasive Services, Jul. 15-20, IEEE Xplore Press, Istanbul, pp: 55-63. DOI: 10.1109/PERSER.2007.4283889 